For Journal of Polymer Science: Polymer Chemistry "Original article"

Revised Feb. 22, 2017

\title{
Hydrolysis of Aromatic Polyurethane in Water under High Pressure of $\mathrm{CO}_{2}$
}

Suguru Motokucho ${ }^{\mathrm{a}}$, Akito Yamaguchi ${ }^{\mathrm{a}}$, Yu Nakayama $^{\mathrm{a}}$, Hiroshi Morikawa ${ }^{\mathrm{b}}$, Hisayuki Nakatani ${ }^{\mathrm{a}}$

${ }^{a}$ Chemistry and Material Engineering Program, Nagasaki University, 1-14, Bunkyo-Machi, Nagasaki-shi, 852-8521, Japan

${ }^{b}$ Department of Applied Chemistry, Kanagawa Institute of Technology, 1030, Shimo-ogino, Atsugi, Kanagawa 243-0292, Japan

Corresponding author Prof. S. Motokucho

Chemistry and Material Engineering Program, Nagasaki University, 1-14, Bunkyo-Machi, Nagasaki-shi, 852-8521, Japan

E-mail:motoku@nagasaki-u.ac.jp

Keywords: Polyurethanes, Recycling, Degradation, Carbon dioxide, Carbonic Acid 


\section{Abstract}

We have demonstrated a hydrolysis reaction of polyurethane (PU) under high pressure of carbon dioxide $\left(\mathrm{CO}_{2}\right)$ in water. We employed the PU sample, poly(methylene bis-(1,4-phenylene)hexamethylene dicarbamate), denoted as M-PU, which was synthesized from 4,4'-diphenyl methane diisocyanate (MDI) and 1,4-butane diol (BD). The optimum hydrolysis reaction condition was $190^{\circ} \mathrm{C}$ under $\mathrm{CO}_{2}$ pressures over 4.1 MPa in water medium, and 93\% hydrolysis of M-PU was achieved. After the reaction, the water-soluble parts were obtained, and isolated by column chromatography. The isolated products were 4,4'-methylenedianiline (MDA) and 1,4-butane diol (BD) which were components of repeating unit of M-PU. In addition, the hydrolysis reaction gave no byproduct. This hydrolysis under high pressure of $\mathrm{CO}_{2}$ with water is a reaction by which M-PU is selectively hydrolyzed into MDA and BD by cleaving urethane linkage. Moreover, the resulting hydrolyzed products were easily obtained by evaporation of aqueous layer after the reaction, indicating an efficient chemical recycling of PU was achieved. 


\section{Introduction}

Polyurethane (PU) is one of the most usable polymeric materials, and widely used for our daily life, i.e., textile, foam, elastomer, adhesive, biomaterial and so on. It has been urgently required that polymeric materials are recycled under safe, efficient and environmental-friendly conditions to realize sustainable society. Among recycling methods of PU materials, chemical recycling is a promising means, and enable the PUs to convert back to starting chemicals. ${ }^{1}$

In general, the processes applicable to chemical recycling of PUs include hydrolysis, aminolysis, alcoholysis, glycolysis, etc. ${ }^{1,2,3}$ However, such processes usually require severe and harsh reaction conditions by using strong acids or bases as catalysts because of inherent chemical stability of urethane linkages. ${ }^{4,5}$ They result in affording complicated reaction mixture containing various kinds of degradation products through the excessive degradation or oxidation etc. ${ }^{1,2}$

The key concept in these recycling is development of catalyst of the hydrolysis, which is safe, cheap, abundant and readily available, easily removable from the reaction mixture, and simple handlings without neutralization of the reaction mixture after the reaction. To avoid excessive degradation of hydrolyzed products, the appropriate catalytic activity is also required for the acid or base catalyst.

On the other hand, it is well-known that $\mathrm{CO}_{2}$, especially under high pressure, effectively gives carbonic acid in situ by reaction with water. ${ }^{6,7}$ This carbonic acid acts as an acid catalyst. ${ }^{8}$ Indeed, this $\mathrm{CO}_{2}$-water system can enhance some organic reactions such as reduction, ${ }^{7}$ hydrolysis, ${ }^{9-11}$ and dehydration. ${ }^{12}$

Previously, we have already reported the hydrolysis of polyurea by using $\mathrm{H}_{2} \mathrm{O}$ with high pressure of $\mathrm{CO}_{2}{ }^{9-11}$ In this hydrolysis reaction, the formed carbonic acid acted as 
an acid catalyst. Besides, this hydrolysis reaction produced the corresponding diamine derived from repeating units of polyurea in an excellent yield. It is noted that the diamine could be easily obtained by evaporation of the aqueous solution in the reaction since this system do not contain additives such as non-volatile acids.

In this paper, we applied this $\mathrm{CO}_{2}$-water system to chemical recycling of $\mathrm{PU}$ by the hydrolysis of the polymer with carbonic acid as a catalyst. As a typical example of PUs, we focused on a representative polyurethane (poly(methylene bis-(1,4-phenylene)hexamethylene dicarbamate): M-PU), which was synthesized from 4,4'-diphenyl methane diisocyanate (MDI) with 1,4-dutane diol (BD). The hydrolysis of M-PU was carried out in an aqueous solution under sub- or super-critical $\mathrm{CO}_{2}$ conditions (below and above 7.3 MPa). Importantly, the system is chemically safe and environmental-friendly because of the components of only $\mathrm{CO}_{2}$ and water with polymers as the substrate. After the reaction, $\mathrm{CO}_{2}$ is vaporized after the reaction vessel is opened, meaning that the system does not require neutralization of the reaction mixture. Therefore, this system will contribute to producing valuable chemicals from polymeric materials. 


\section{EXPERIMENTAL}

Materials. 1,4-butanediol (BD, WAKO Chemicals Co., Ltd., Osaka, Japan), and N,N-dimethylformamide (DMF, WAKO Chemicals Co., Ltd., Osaka, Japan) were used after distillation under reduced pressure. 4,4'-Diphenylmethane diisocyanate (MDI, Nippon Polyurethane Industry Co. Ltd., Yamaguchi, Japan), N,N-dimethylacetamide (DMAc, WAKO Chemicals Co., Ltd., Osaka, Japan) and dimethyl sulfoxide (DMSO, WAKO Chemicals Co., Ltd., Osaka, Japan) were used as received.

Characterization. IR spectrum was recorded with Bio-Rad Laboratories FTS $3000 \mathrm{MXN}$, and the values are given in $\mathrm{cm}^{-1}$. ${ }^{1} \mathrm{H}$-nuclear magnetic resonance $\left({ }^{1} \mathrm{H}-\mathrm{NMR}\right)$ spectra of products were recorded on a JNM-GX400 at $400 \mathrm{MHz}$. The number- and weight-average molecular weights $\left(M_{\mathrm{n}}\right.$ and $M_{\mathrm{w}}$, respectively) and polydispersity index $\left(M_{\mathrm{w}} / M_{\mathrm{n}}\right)$ were estimated by gel permeation chromatography (GPC) on a polystyrene gel column (Shimadzu Shim-pack GPC-802) using a Shimadzu HPLC 20AD pump system equipped with a refractive index detector with a DMF eluent at a flow rate of $1.0 \mathrm{~mL} / \mathrm{min}$, which was calibrated by polystyrene standards. Thermal analyses were measured with RIGAKU Thermos Plus DSC8230HT and TG8120.

Preparation of poly(methylene bis-(1,4-phenylene)hexamethylene dicarbamate (M-PU). To a solution of MDI (8.88 g, $35.5 \mathrm{mmol})$ in DMF (70 mL), BD (3.26 g, $36.2 \mathrm{mmol}$ ) was added at $60{ }^{\circ} \mathrm{C}$, and the reaction temperature was raised up to $80{ }^{\circ} \mathrm{C}$ for 1.5 hours. The consumption of isocyanate group was monitored by titration with dibutylamine. ${ }^{13}$ The reaction mixture was poured into methanol and then the white solid was precipitated. The resulting precipitate was filtrated and washed with methanol by a Soxhlet extractor, and dried in vacuo to give a white solid of M-PU ( 8.75 
g, 72.1\%). The obtained M-PU was insoluble in several solvents, such as methanol, ethanol, toluene, chloroform, hexane, ethyl acetate and water, and was soluble in high polar amide or sulfoxide solvent such as DMF, DMAc and DMSO.

$\operatorname{IR}(\mathrm{KBr})$ : $3321\left(\mathrm{v}(\mathrm{N}-\mathrm{H})_{\text {H-bond }}\right), 1701\left(\mathrm{v}(\mathrm{C}=\mathrm{O})_{\text {H-bond }}\right), 1528(\mathrm{v}(\mathrm{C}-\mathrm{N}),(\mathrm{N}-\mathrm{H}))$ and 1227 $(v(\mathrm{C}-\mathrm{O})) \mathrm{cm}^{-1} .{ }^{1} \mathrm{H}-\mathrm{NMR}(400 \mathrm{MHz}$, in DMSO-d6, $\delta: \mathrm{ppm}): 9.53(\mathrm{~s}, 2 \mathrm{H}), 7.35-7.37(\mathrm{~d}$, $4 \mathrm{H}), 7.10-7.08(\mathrm{~d}, 4 \mathrm{H}), 4.10(\mathrm{~s}, 4 \mathrm{H}), 3.78(\mathrm{~s}, 2 \mathrm{H}), 1.70(\mathrm{~s}, 4 \mathrm{H}) . \quad T_{\mathrm{d} 5}=293{ }^{\circ} \mathrm{C}$.

General procedure of hydrolysis of M-PU. $\quad 0.5 \mathrm{~g}$ of M-PU and $20 \mathrm{~mL}$ of water were placed into a $200 \mathrm{~mL}$ stainless autoclave equipped with a pressure gauge followed by the introduction of appropriate amount of liquid $\mathrm{CO}_{2}$. Then, the autoclave was heated using a band heater under high pressure of $\mathrm{CO}_{2}(8.0 \mathrm{MPa})$. After a given reaction time, the reactor was rapidly immersed into an ice bath to cool down to room temperature. A valve of the reactor was opened to release pressured $\mathrm{CO}_{2}$. Finally, the reaction mixture was collected from the reactor and separated to the filtrate and residual water-insoluble parts with a filter paper. The obtained residual water-insoluble parts was washed with water and methanol before dried under vacuum and weighed. The filtrate was concentrated by the rotary evaporator and gave the residual oil containing solid. The residual oil containing solid was weighed after drying. The residual water-insoluble parts and residual oil containing solid, the latter of which was obtained from the filtrate, were analyzed by ${ }^{1} \mathrm{H}-\mathrm{NMR}$ spectroscopy in DMSO- $d 6$. 


\section{RESULTS AND DISCUSSION}

Preparation of poly(methylene bis-(1,4-phenylene)hexamethylene dicarbamate) (M-PU)

Scheme 1 shows preparation scheme of representative polyurethane (M-PU). The M-PU was obtained by the polyaddition reaction of $4,4^{\prime}$-diphenyl methane diisocyanate $(\mathrm{MDI})$ and 1,4-butane diol $(\mathrm{BD})$ with molar ratio of $[\mathrm{BD}]_{0} /[\mathrm{MDI}]_{0}=1.02 . \quad \mathrm{A}$ small excess of BD was used for synthesis of M-PU having hydroxyl group at the terminal unit. The polyaddition at $80{ }^{\circ} \mathrm{C}$ was quenched within $1.5 \mathrm{~h}$. We confirmed the consumption of isocyanate group by titration with dibutylamine. ${ }^{13}$ The obtained M-PU was characterized by Fourier transform infrared (FT-IR), ${ }^{1} \mathrm{H}-\mathrm{NMR}$ spectroscopic analyses and gel permeation chromatography (GPC) measurements. 5\% weight loss temperature $\left(T_{\mathrm{d} 5}\right)$ of $\mathrm{M}-\mathrm{PU}$ was $293{ }^{\circ} \mathrm{C}$ with the TGA analysis.

Figure 1 shows ${ }^{1} \mathrm{H}-\mathrm{NMR}$ spectrum of M-PU in DMSO-d6. In the spectrum, signals $a$ and $b$ at 7.08 7.10 and 7.35 7.37 ppm,,$e$ and $f$ at 9.53, 4.10 and 1.70 ppm, $d$ at $3.78 \mathrm{ppm}$, and $j$ at $3.58 \mathrm{ppm}$ were assigned to 2,3-position of aromatic group, - $\mathrm{NH}$-, $\alpha-\mathrm{CH}_{2}$ (-NH-CO-OCH $\left.\mathrm{CH}_{2}-\right)$ and $\beta-\mathrm{CH}_{2}\left(-\mathrm{NH}-\mathrm{CO}-\mathrm{OCH}_{2} \mathrm{CH}_{2}-\right)$ of urethane bond, methylene group (-Ph- $\left.\mathrm{CH}_{2}-\mathrm{Ph}-\right)$ and terminal unit of $-\mathrm{CH}_{2} \mathrm{OH}$, respectively. No signals attributable to aniline terminal units and urea groups were observed. These results suggested that the obtained M-PU had a desired chemical structure bearing hydroxyl terminal units. The number-average molecular weight $\left(M_{\mathrm{n}}\right)$ of M-PU was estimated from the integral ratio of signals $j$ to $f$. (see Table 1)

\section{Hydrolysis Reaction of M-PU and Identification of Reaction Products}


The hydrolysis of M-PU $(0.50 \mathrm{~g})$ in water $(20 \mathrm{~mL})$ was carried out under high pressure $\mathrm{CO}_{2}$ of $8.0 \mathrm{MPa}$ at $190^{\circ} \mathrm{C}$ for $24 \mathrm{~h}$.

The $\mathrm{pH}$ value of the aqueous solution under high pressure of $\mathrm{CO}_{2}$ was studied in ref. $6,14-17$, especially ref.16 in detail. However, in all of them, the value was measured at lower than $150{ }^{\circ} \mathrm{C}$ (not at $190{ }^{\circ} \mathrm{C}$ ). In this study, we did not measure the value because the measurement needs special technique and equipment. In the previous study ${ }^{16}$, it was reported that a $\mathrm{pH}$ value under $9 \mathrm{MPa} \mathrm{CO}_{2}$ at $150^{\circ} \mathrm{C}$ is around 3.5 , and it decreases with increase in $\mathrm{CO}_{2}$ pressure and decrease in the temperature. According to the study, the value under our condition of $8 \mathrm{MPa}^{\mathrm{CO}} \mathrm{O}_{2}$ at $190^{\circ} \mathrm{C}$ was expected to be 3.5 or a little higher than it.

The temperature was much lower than $T_{\mathrm{d} 5}\left(293{ }^{\circ} \mathrm{C}\right)$, and then thermal decomposition was negligible in the reaction. After the reaction, the reaction mixture was divided into the residual water-insoluble parts and the filtrate as water-soluble parts. First, the residual water-insoluble parts was washed with water and methanol, and dried in vacuo at $60{ }^{\circ} \mathrm{C}$ for $24 \mathrm{~h}$. White powder of $0.08 \mathrm{~g}$ was obtained at this reaction condition.

Figure 2 shows ${ }^{1} \mathrm{H}-\mathrm{NMR}$ spectrum of the residual water-insoluble parts in DMSO-d6. In contrast to M-PU, in spectrum for the residual water-insoluble parts, one set of signals $(\mathrm{a} \sim \mathrm{k})$ were observed at same values of chemical shifts to those of M-PU. This means the chemical structure of the residual water-insoluble parts is same to repeating units of M-PU. Additionally, some new signals appeared at 6.42-6.44 and 6.79-6.81 ppm ( $A$ and $B), 4.81 \mathrm{ppm}(m), 3.63 \mathrm{ppm}\left(d^{\prime}\right)$. These new signals were assigned to aromatic protons of aniline unit, - $\mathrm{NH}_{2}$ group, and methylene protons (-Ph- $\mathrm{CH}_{2}-\mathrm{Ph}-\mathrm{NH}_{2}$ ), all of which were located at the terminal, respectively. 
These NMR analyses suggest that the hydrolysis of main chain of M-PU took place under high pressure of $\mathrm{CO}_{2}$, and produced a partially hydrolyzed $\mathrm{PU}$ oligomer bearing not only hydroxyl units but also aniline units at the terminal units as residual water-insoluble parts after the hydrolysis.

Figure 3 shows GPC profiles of originated M-PU (solid line) and the obtained residual water-insoluble parts (dashed line). Almost unimodal profiles were observed in the elution peaks for both samples. Besides, for residual water-insoluble parts, there were also shoulders in addition to the main peak. It suggests that the hydrolysis is not a complete random process to some extent. The values of $M_{\mathrm{n}}$ (estimated from NMR and GPC) and $M_{\mathrm{w}} / M_{\mathrm{n}}$ for originated M-PU and the residual water-insoluble parts are summarized in Table 1. The $M_{\mathrm{n}}$ of the residual water-insoluble parts was obviously lower than that of M-PU. The comparison of molecular weight for both samples indicates that the residual water-insoluble parts had a low molecular weight. Decrease in $M_{\mathrm{n}}$ for the residual water-insoluble parts from the originated M-PU is consistent with change in the NMR spectra. On the basis of the two experimental data, certainly the obtained residual water-insoluble parts have same repeating units to that of M-PU, and have hydroxyl unit or aniline unit or both at the terminal in the structure. And molecular weight of M-PU decreased with this hydrolysis reaction.

Next, the filtrate after the hydrolysis was concentrated by the rotary evaporator and gave $0.36 \mathrm{~g}$ of residual oil containing solid as water soluble parts. The obtained oil containing solid was purified by column layer chromatography. It was found that the obtained compound was a mixture of 4,4'-methylenedianiline (MDA) and 1,4-butanediol (BD). These products were components of repeating unit of M-PU. Figure 4 shows ${ }^{1} \mathrm{H}-\mathrm{NMR}$ spectrum of the oil containing solid before purification. 
Observed signals $A$ and $B$ at $6.42-6.44$ and $6.79-6.81 \mathrm{ppm}, m$ at $4.79 \mathrm{ppm}$, and $d$ at 3.63 ppm were assigned to protons due to a set of MDA as shown in Figure 4. Similarly, signals $k, j$ and $i$ at 4.47, 3.42 and 1.45 ppm were assigned to protons due to a set of BD. Additionally, no other signals were observed in this oil containing solid. It suggested that the hydrolysis of M-PU under high pressure of $\mathrm{CO}_{2}$ proceeded with high selectivity into raw materials.

The degree of hydrolysis of M-PU was estimated from following equation:

Degree of hydrolysis $(\%)=([$ Weight of starting M-PU]-[Weight of water-insoluble residue] / [Weight of starting M-PU]) $\times 100$

In terms of the degree of hydrolysis of M-PU, effects of the reaction conditions were evaluated.

In all of the reactions, $20 \mathrm{~mL}$ water and $0.5 \mathrm{~g}$ M-PU were used.

\section{Effect of reaction time and $\mathrm{CO}_{2}$ on the degree of hydrolysis of M-PU}

The hydrolysis reaction of M-PU was carried out at $190{ }^{\circ} \mathrm{C}$ under $8.0 \mathrm{MPa}$ of $\mathrm{CO}_{2}$ as shown in Figure 5. Under $8.0 \mathrm{MPa}$ of $\mathrm{N}_{2}$ atmosphere instead of $\mathrm{CO}_{2}$, degree of hydrolysis of M-PU did not reach 5\% even after $24 \mathrm{~h}$. This result is within the range of technical loss. On the other hand, under $\mathrm{CO}_{2}$, the reaction proceeded to give $14 \%$ of hydrolysis for 1 hour. The degree of hydrolysis of M-PU increased smoothly with increasing reaction time, resulting in excellent hydrolyzed degrees of $65 \%$ and $93 \%$ for 
$12 \mathrm{~h}$ and $52 \mathrm{~h}$, respectively. It was concluded that the hydrolysis of M-PU was accelerated under high pressure of $\mathrm{CO}_{2}$.

\section{Effect of temperature on the degree of hydrolysis of M-PU}

This hydrolysis reaction was applied to each temperature to investigate the effect of the reaction temperature on this reaction. Figure 6 shows the relationship between temperature and degree of hydrolysis of M-PU for $12 \mathrm{~h}$ under $8.0 \mathrm{MPa}$ of $\mathrm{CO}_{2}$. From 120 to $160{ }^{\circ} \mathrm{C}$, the degree of hydrolysis of M-PU was below $10 \%$. Over $160{ }^{\circ} \mathrm{C}$, the degree of hydrolysis was smoothly increased with increasing temperature. At 180 and $190{ }^{\circ} \mathrm{C}$, the degree rose remarkably to 43 and $65 \%$, respectively. It was found that the value of natural logarithm of the degree, $\ln ($ conversion $)$, was linearly proportioned to the reaction temperature (data is not shown). It means the hydrolysis proceeded dependent on the reaction temperature, and an adequate degree of hydrolysis was obtained above $160{ }^{\circ} \mathrm{C}$ under this condition.

\section{Pressure of $\mathrm{CO}_{2}$}

Figure 7 shows the relationship between pressure of $\mathrm{CO}_{2}(2.0-14.2 \mathrm{MPa})$ and degree of hydrolysis of M-PU at $190{ }^{\circ} \mathrm{C}$ for $2 \mathrm{~h}$. The degree of hydrolysis of M-PU was increased with increasing pressure from 2.0 to 4.1 $\mathrm{MPa}$. It reached a maximum value at 4.1 $\mathrm{MPa}$, which is below critical pressure of $\mathrm{CO}_{2}$ at $190{ }^{\circ} \mathrm{C}$. The increasing of the degree until 4.1 MPa might be from the increased formation of carbonic acid due to the dependence of $\mathrm{CO}_{2}$ pressure. On the other hand, the opposing behavior over 4.1 $\mathrm{MPa}$ might come from the decreased dissociation or transfer or both of protons from carbonic acid due to the decreased polarity of the solvent. ${ }^{18,19}$ The similar behavior 
dependent on pressure of $\mathrm{CO}_{2}$ was observed in hydrolysis of polyurea ${ }^{9-11}$ and dehydration of fructose ${ }^{12}$ in $\mathrm{CO}_{2}$-water system in our previous works.

\section{Mechanism of Hydrolysis Reaction}

Scheme 2 illustrates a plausible mechanism for this hydrolysis reaction of M-PU. First, carbonic acid was formed by the reaction of $\mathrm{CO}_{2}$ with $\mathrm{H}_{2} \mathrm{O} .{ }^{6,14-17}$ The carbonic acid could release protons by the dissociation of in situ reaction. Then the protons activated carbonyl group of urethane one [IM-A]. The activated carbonyl carbon of urethane group reacted with $\mathrm{H}_{2} \mathrm{O}$ to form oxonium cationic intermediate [IM-B]. The intermediate released proton and gave carbamic acid group and hydroxyl ones (hydroxyl terminal). The carbamic acid group gave amino group (aniline terminal) with releasing $\mathrm{CO}_{2}$.

When the hydrolysis condition of M-PU is $8.0 \mathrm{MPa}$ at $190{ }^{\circ} \mathrm{C}$ for $24 \mathrm{~h}$, the composition of [aniline terminal unit]:[hydroxyl terminal unit] in hydrolyzed water insoluble solid part and [MDA]:[BD] in the filtrate were estimated from the integral ratio of signals $A$ to $i$, in each ${ }^{1} \mathrm{H}-\mathrm{NMR}$ spectrum. The value of [aniline terminal unit]:[hydroxyl terminal unit] and [MDA]:[BD] were 81.6:18.4 and 17.9:82.1, respectively. This result suggested that the hydroxyl terminal is more hydrolysable than aniline terminal in this hydrolysis reaction.

All samples hydrolyzed under varying examined conditions showed similar trends, that is, the partially hydrolyzed M-PU (the residual water-insoluble parts) was rich in the ratio of aniline (MDA-derived) terminal unit whereas the filtrate was rich in that of BD. These results indicated that, in this pathway (Scheme 2), urethane moiety close to the hydroxyl (BD unit) terminal unit is more hydrolysable than that close to aniline 
(MDA unit) terminal unit, which caused the richness of BD as the hydrolyte in the filtrate. The difference of the hydrolytic behavior of the two terminal units would be explained by the expectation that the hydroxyl unit at the terminal is more hydrophilic than aniline units bearing phenyl rings, allowing the neighboring urethane moiety to contact with water and protons. We expected that, even if amino group is protonated by carbonic acid in water to form ammonium one, the access of water to urethane group would be inhibited by highly hydrophobic bis-phenyl methane group as the spacer between the two groups.

\section{CONCLUSION}

We have successfully demonstrated the efficient promotion of hydrolysis of M-PU in high-temperature water under high pressure of $\mathrm{CO}_{2}$ which are nonhazardous in nature. The effects of such reaction condition as reaction time, temperature, and $\mathrm{CO}_{2}$ pressure were clarified. Important advantage of this process is that, after the hydrolysis, it did not require neutralization of the reaction mixture and further purification to obtain the corresponding hydrolytes MDA and BD with high selectivity. These advantageous features cannot be attained by methods with various kinds of non-volatile acids.

The process is a general method for the hydrolysis of urethane linkage with carbonic acid from the reaction of $\mathrm{CO}_{2}$ with $\mathrm{H}_{2} \mathrm{O}$. We believe this chemical recycling of $\mathrm{PU}$ is in great practical value because of the system consists of $\mathrm{CO}_{2}$ and water without any nonvolatile additives, leading to be a safety, efficient, cheap, and easily handling method. 


\section{ACKNOWLEDGEMENTS}

This work was supported by the Ministry of Education, Culture, Sports, Science and Technology KAKENHI Grant-in-Aid for Young Scientists (B) Grant Number 25810078 and Japan Society for the Promotion of Science is funding agency of Japanese Ministry of Science Grant-in-Aid for "Program for Advancing Strategic International Networks to Accelerate the Circulation of Talented Researchers". And the author is sincerely grateful to Dr. Toshio Inoue, JX Nippon Oil \& Energy Corporation. 


\section{REFERENCES AND NOTES}

1. J. Scheirs, Polymer Recycling; Wiley: New York, 1998; Chapter 10.

2. K. M. Zia, H. N. Bhatti, I. A. Bhatti, React. Funct. Polym. 2007, 67, 675-692.

3. L. Liu, L. Tang, Z. Zhu, Y. Ni and Y. Wu, Proceedings of MATEC Web of Conferences 2016, 64, 02002.

4. K. Sato, K. Sumitani, Jpn. Pat. 1995, 7,309,810 TEIJIN Limited.

5. K. Ishihara, K. Ishida, Jpn. Pat. 1999, 11,302,227 TEIJIN Limited.

6. K. L. Toews, R. M. Shroll, C. M. Wai, N. G. Smart, Anal. Chem., 1995, 67, 4040-4043.

7. K. V. West, C. Wheeler, J. P. McCarney, K. N. Griffith, D. Bush, C. L. Liotta, C. A. Eckert, J. Phys. Chem. A. 2001, 105, 3947-3948.

8. G. Li, H. Jiang, J. Li, Green Chem. 2001, 3, 250-251.

9. M. Furukawa, K. Kojio, S. Motokucho, PCT pat. 2009, WO 2009130842.

10. M. Furukawa, K. Kojio, S. Motokucho, PCT pat. 2010, WO 2010125743.

11. S. Motokucho, T. Matsumoto, Y. Nakayama, R. Horiuchi, H. Morikawa, and H. Nakatani, Polym. Bull., 2017, 74(3), 615-623.

12. S. Motokucho, H. Morikawa, H. Nakatani, B. A. J. Noordover, Tetrahed. Lett., 2016, 57, 4742-4745. 
13. A. V. Mayer, Annual book of ASTM standards, 2010, D5155.

14. C. Roosen, M. Ansorge-Schumacher, T. Mang, W. Leitner, L. Greiner, Green Chem., 2007, 9, 455-458.

15. X. X. Wang, H. Fu, D.M. Du, Z. Y. Zhou, A. G. Zhang, C. F. Su, K. F. Ma, Chem. Phys. Lett., 2008, 460, 339-342.

16. C. Peng, J. P. Crawshaw, G. C. Maitland, J. P. M. Trusler, D. Vege-Maza, J. Supercrit. Fluids, 2013, 82, 129-137.

17. M. A. Pigaleva, I. V. Elmanovich, Y. N. Koonevich, M. O. Gallyamov, A. M. Muzafarov, RSC Adv., 2015, 5, 103573-103608.

18. X. Xie, C. L. Liotta, and C. A. Eckert, Ind. Eng. Chem. Res., 2004, 43, 2605-2609.

19. R. Ma, A.-H. Liu, C.-B. Huang, X.-D. Li and L.-N. He, Green Chem. 2013, 15, 1274-1279. 
Table 1. $M_{\mathrm{n}}$ and $M_{\mathrm{w}} / M_{\mathrm{n}}$ of M-PU and residual water-insoluble parts (hydrolyzed M-PU)

\begin{tabular}{crrc}
\hline & $M_{\mathrm{n}, \mathrm{NMR}}$ & $M_{\mathrm{n}, \mathrm{GPC}}$ & $M_{\mathrm{w}} / M_{\mathrm{n}}$ \\
\hline M-PU & 6,400 & 27,000 & 1.72 \\
hydrolyzed M-PU & 1,200 & 8,500 & 4.42 \\
\hline
\end{tabular}


Figure 1. ${ }^{1} \mathrm{H}-\mathrm{NMR}$ spectrum of synthesized representative polyurethane (M-PU) in DMSO- $d_{6}$.

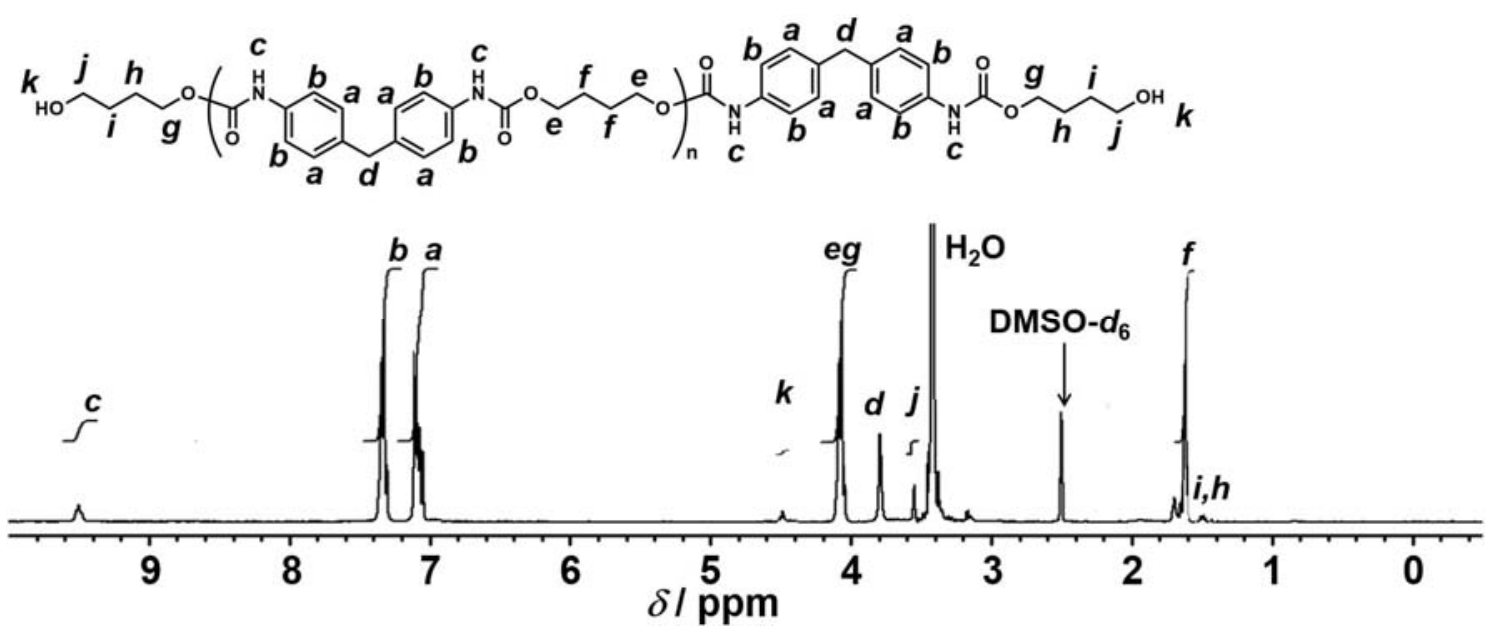


Figure 2. ${ }^{1} \mathrm{H}-\mathrm{NMR}$ spectrum of residual water-insoluble parts (hydrolyzed M-PU) in DMSO- $d_{6}$.
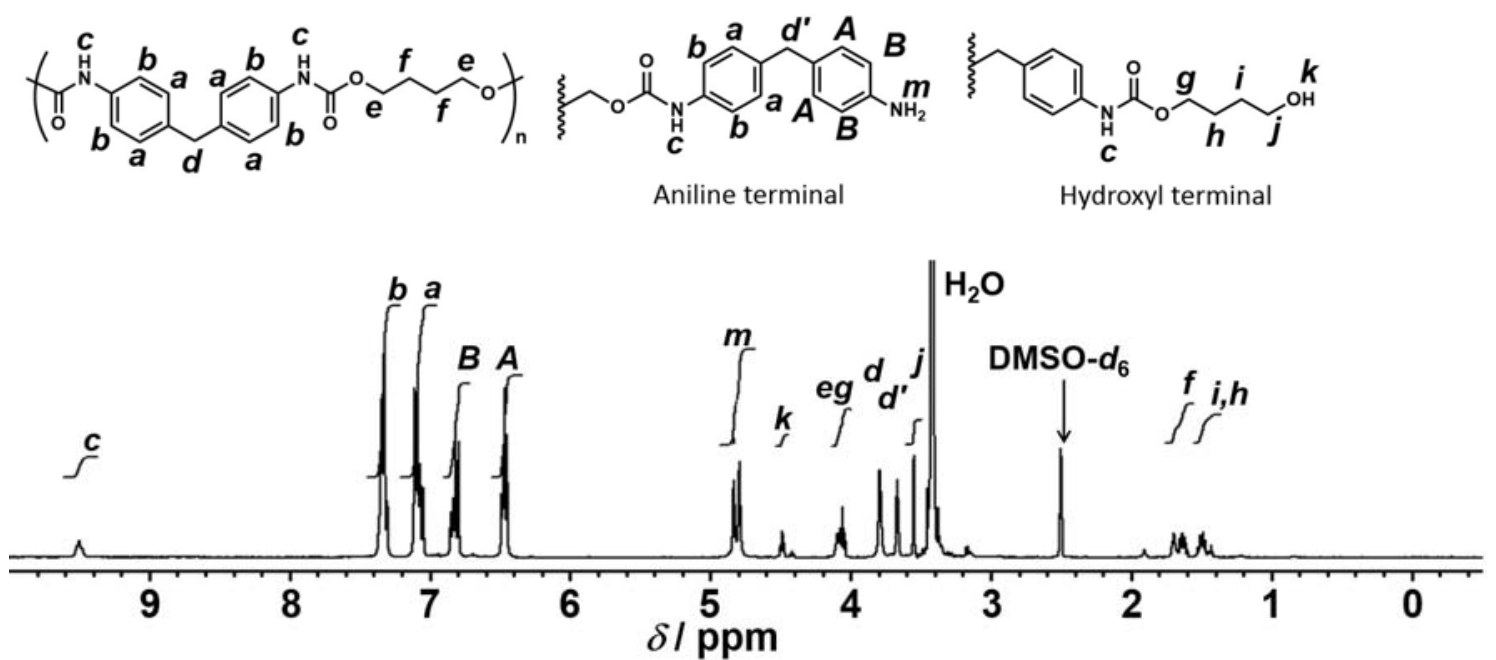
Figure 3. GPC profiles of M-PU (solid line) and residual water-insoluble parts (hydrolyzed M-PU; dashed line).

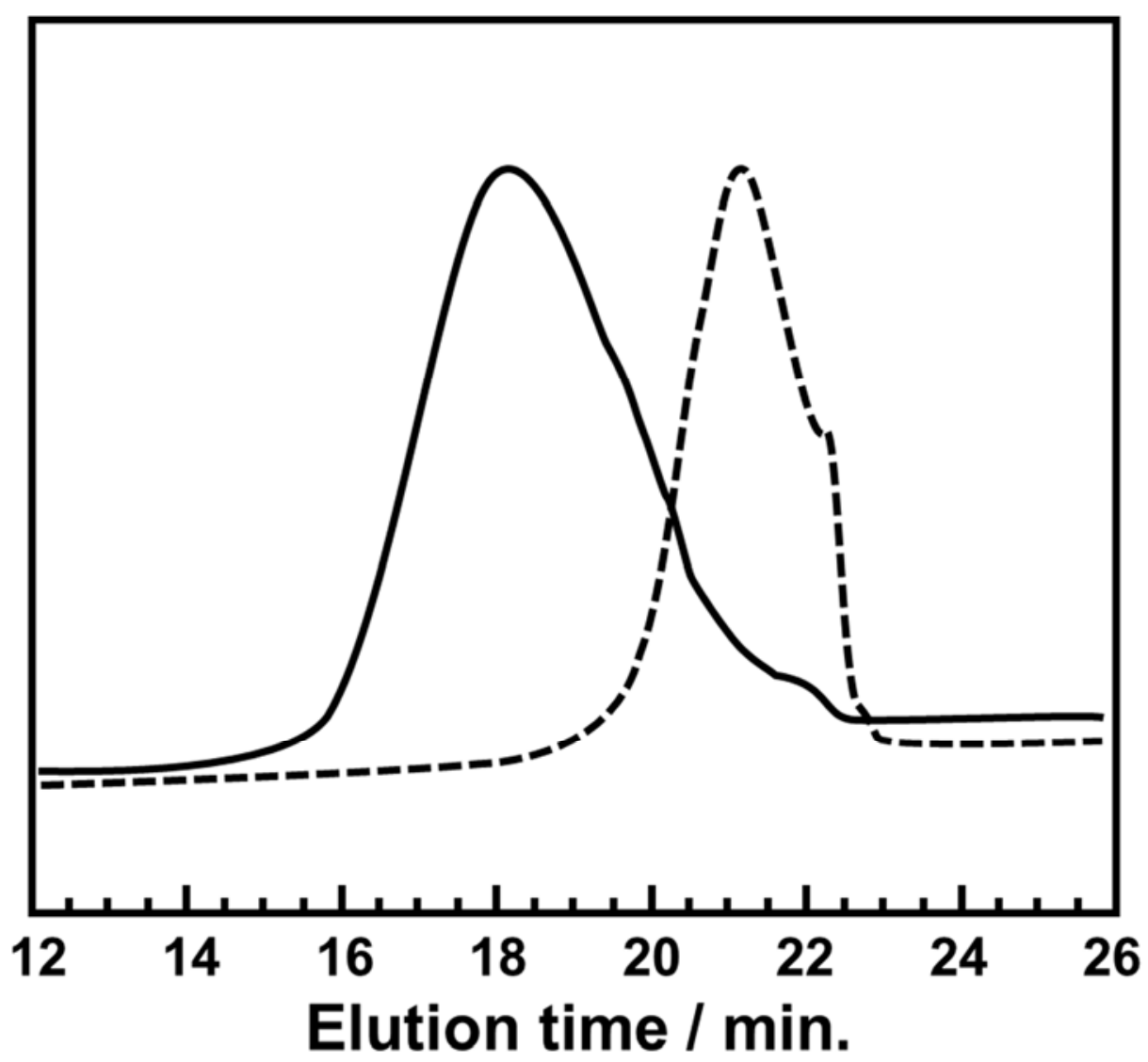


Figure 4. ${ }^{1} \mathrm{H}-\mathrm{NMR}$ spectrum of the filtrate as sluggish compound in DMSO-d6.

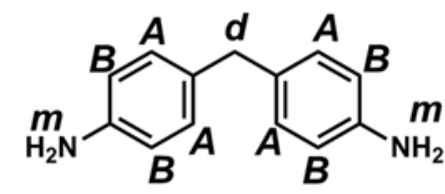<smiles>[R]OC(C)C(C)C(C)C(C)O</smiles>

MDA

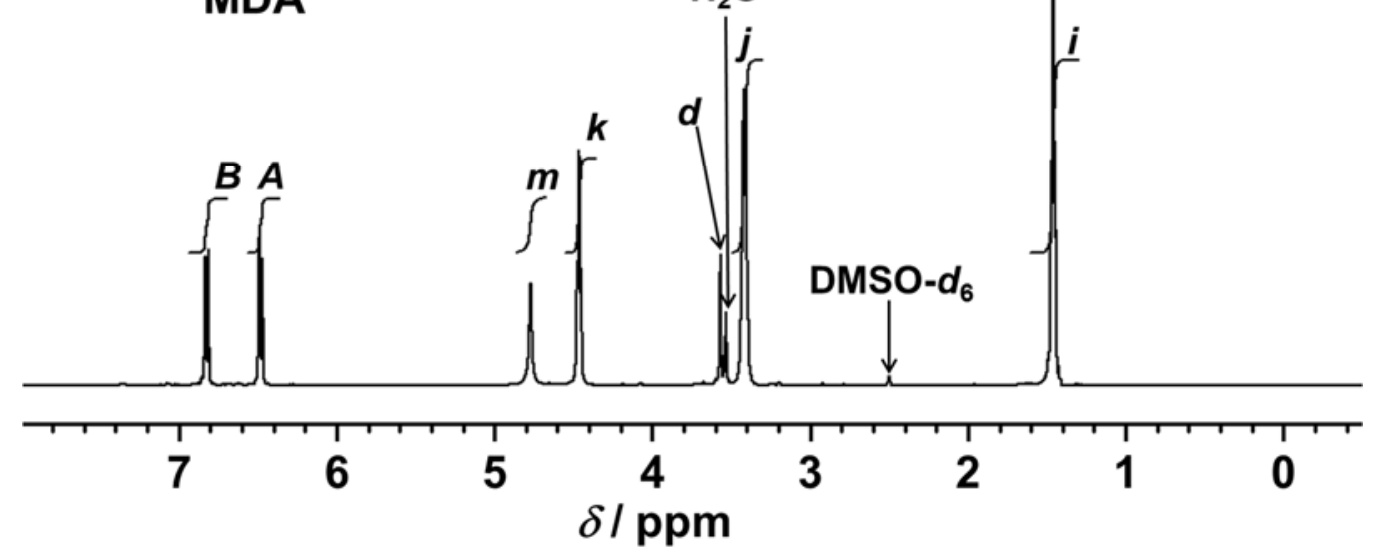


Figure 5. Changes of degree of hydrolysis with reaction time at $190{ }^{\circ} \mathrm{C}$ under $8.0 \mathrm{MPa}$ of $\mathrm{CO}_{2}$.

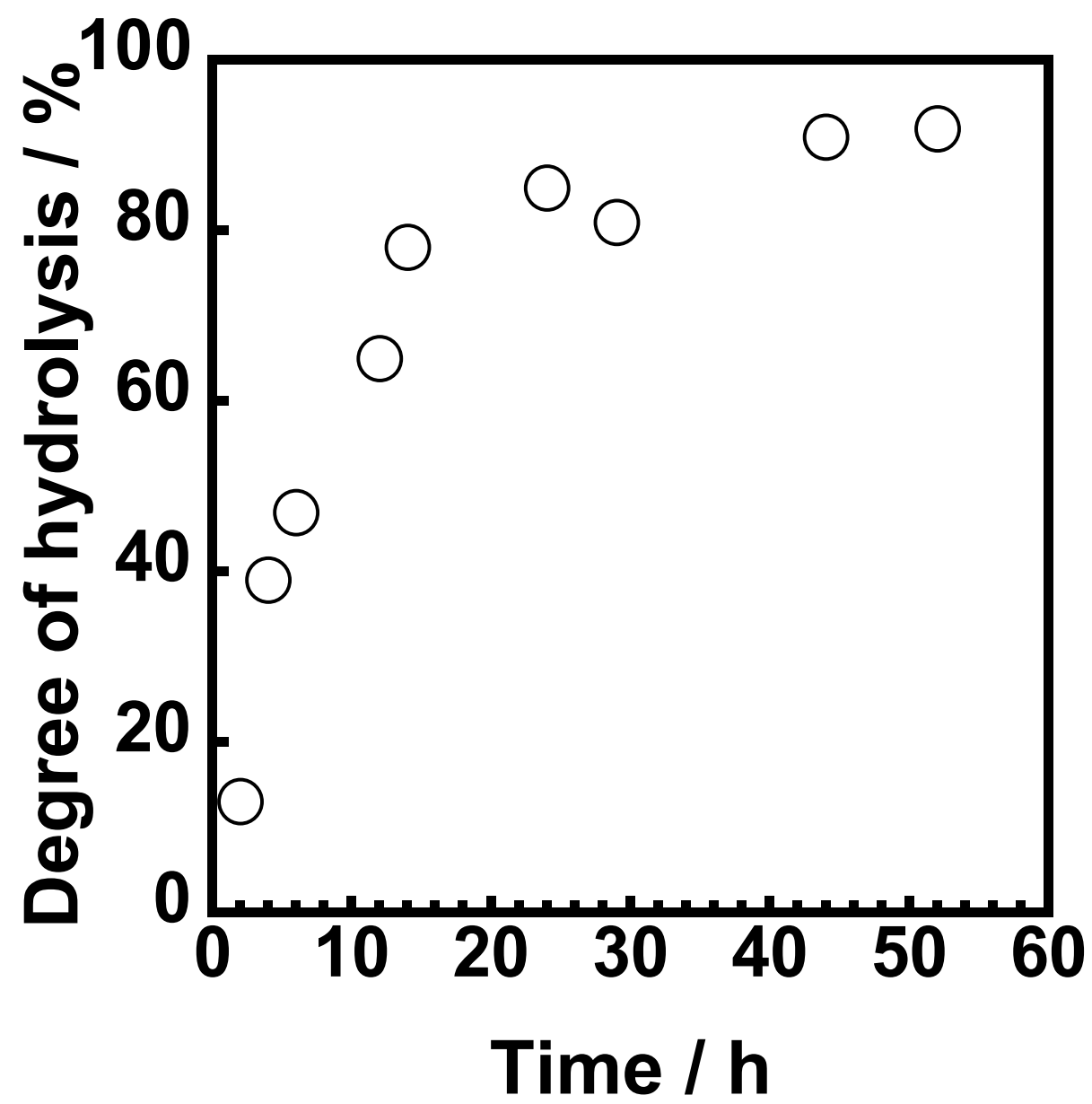


Figure 6. Relationship between reaction temperature and degree of hydrolysis of M-PU for $12 \mathrm{~h}$ under $8.0 \mathrm{MPa}$ of $\mathrm{CO}_{2}$.

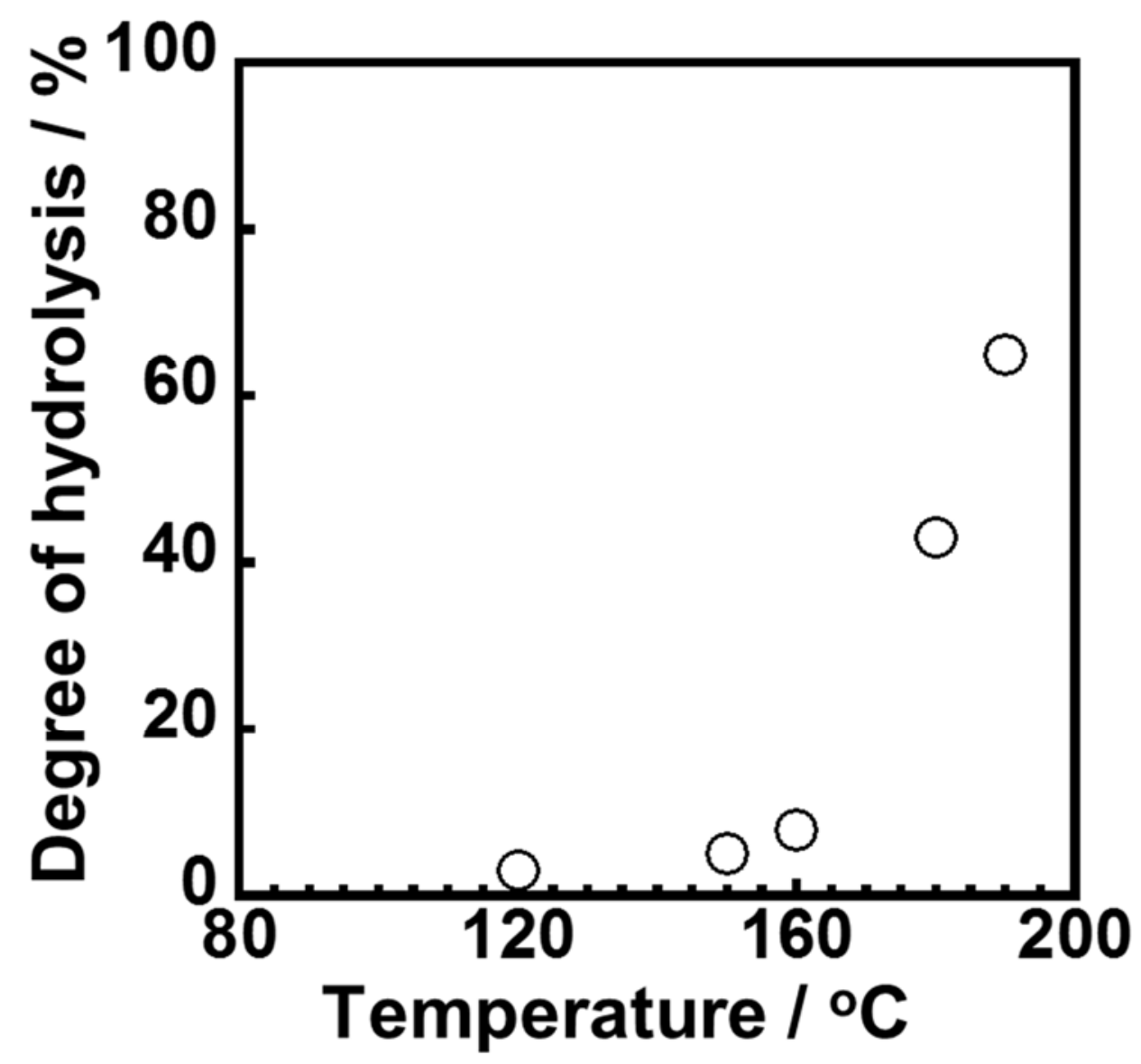


Figure 7. Pressure dependence of the degree of hydrolysis of M-PU at $190{ }^{\circ} \mathrm{C}$ for $2 \mathrm{~h}$.

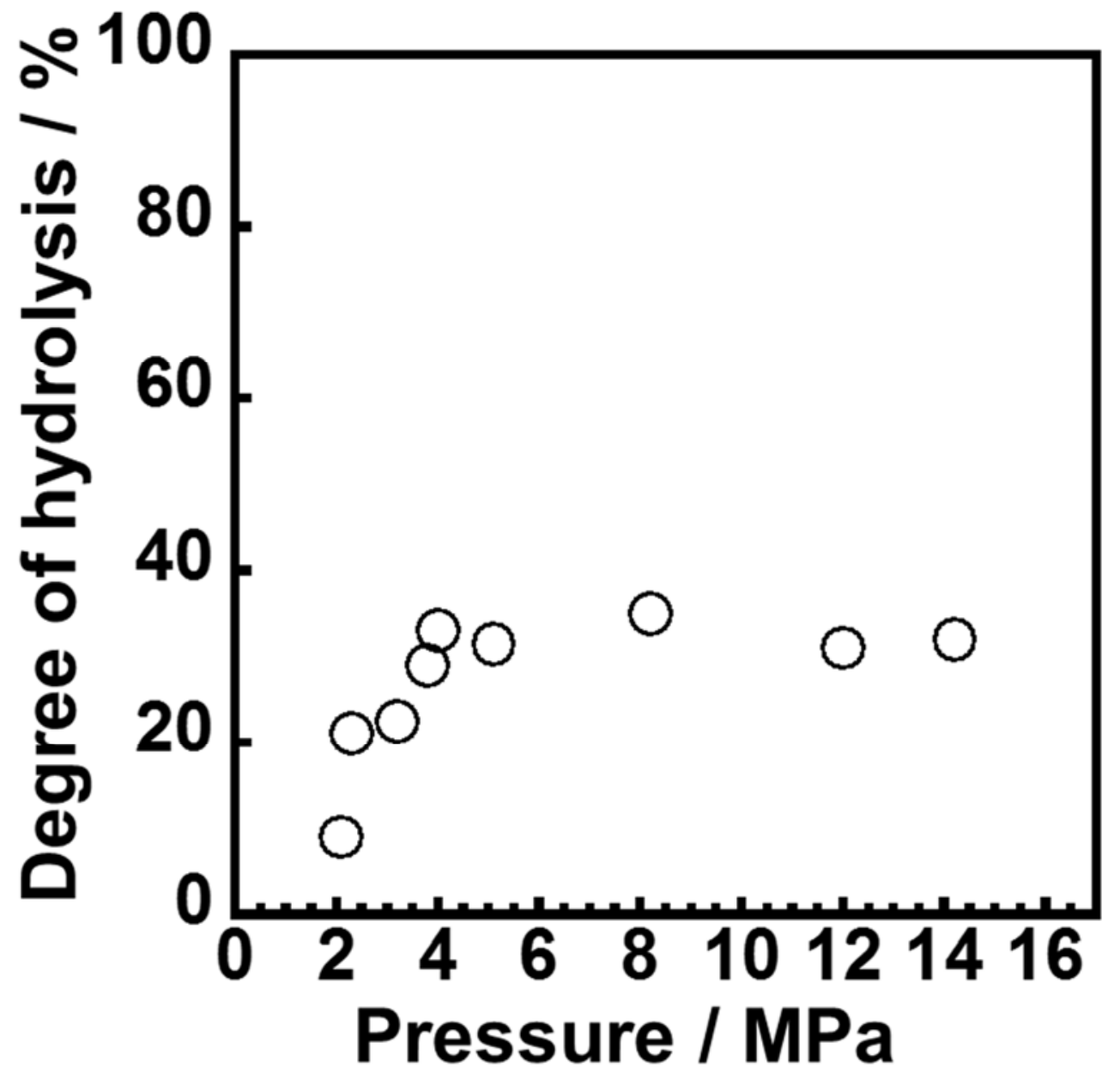


Scheme 1. Preparation scheme of representative polyurethane (M-PU)

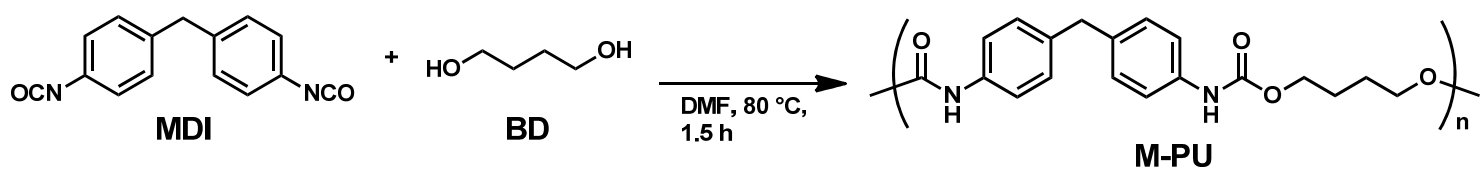


Scheme 2. Illustration of plausible mechanism of hydrolysis of M-PU.
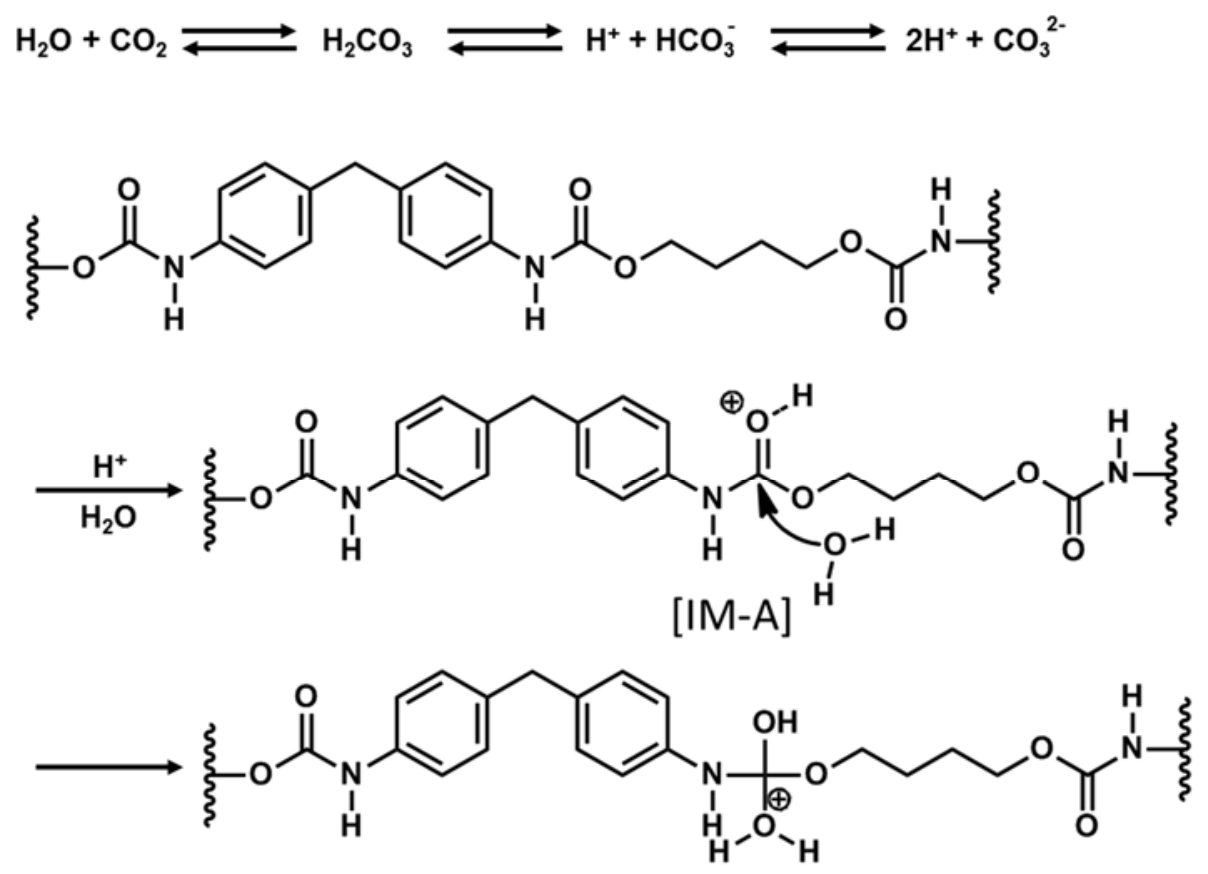

[IM-B]

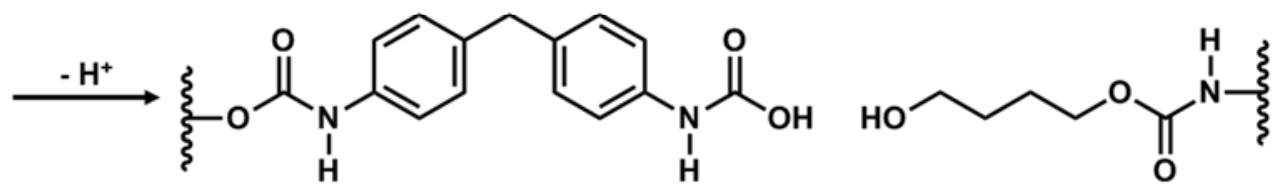

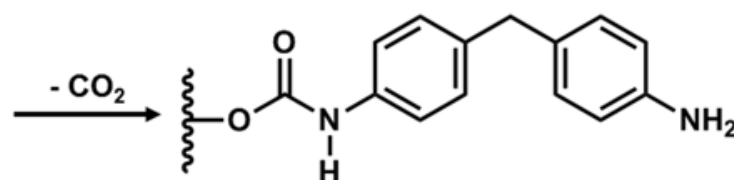

Aniline terminal<smiles>CC(C)NC(=O)OCCCCO</smiles>

Hydroxyl terminal 


\section{GRAPHICAL ABSTRACT}

Hydrolysis of Aromatic Polyurethane in Water under High Pressure of $\mathrm{CO}_{2}$

Suguru Motokucho, Akito Yamaguchi, Yu Nakayama, Hiroshi Morikawa, Hisayuki Nakatani

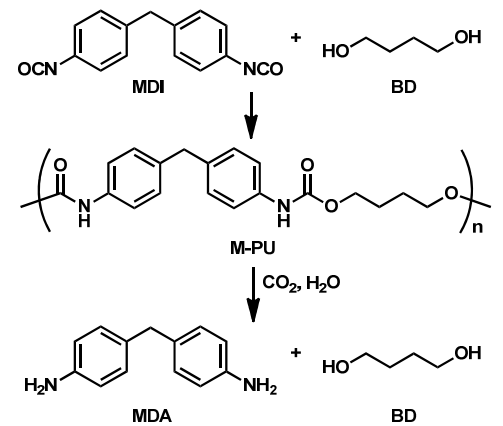

We have demonstrated a hydrolysis reaction of polyurethane (M-PU) under high pressure of carbon dioxide $\left(\mathrm{CO}_{2}\right)$ in water. The optimum hydrolysis reaction condition was $190^{\circ} \mathrm{C}$ under $\mathrm{CO}_{2}$ pressures over 4.1 $\mathrm{MPa}$ in water medium, and $93 \%$ hydrolysis of $\mathrm{M}-\mathrm{PU}$ was achieved. This hydrolysis under high pressure of $\mathrm{CO}_{2}$ with water is a reaction by which M-PU is selectively hydrolyzed into consisted chemicals of repeating units of $\mathrm{M}-\mathrm{PU}$ by cleaving urethane linkage. 